\title{
Front Matter: Volume 6618
}

, "Front Matter: Volume 6618," Proc. SPIE 6618, O3A: Optics for Arts, Architecture, and Archaeology, 661801 (21 April 2009); doi:

10.1117/12.746775

SPIE. Event: Optical Metrology, 2007, Munich, Germany 


\title{
PROCEEDINGS OF SPIE
}

\section{O3A: Optics for Arts, Architecture, and Archaeology}

\author{
Costas Fotakis \\ Luca Pezzati \\ Renzo Salimbeni \\ Editors
}

20-22 June 2007

Munich, Germany

Sponsored by

SPIE Europe

Cooperating Organizations

EOS-European Optical Society

WLT-Wissenschaftliche Gesellschaft Lasertechnik e.V. (Germany)

Published by

SPIE

Volume 6618 
The papers included in this volume were part of the technical conference cited on the cover and title page. Papers were selected and subject to review by the editors and conference program committee. Some conference presentations may not be available for publication. The papers published in these proceedings reflect the work and thoughts of the authors and are published herein as submitted. The publisher is not responsible for the validity of the information or for any outcomes resulting from reliance thereon.

Please use the following format to cite material from this book:

Author(s), "Title of Paper," in O3A: Optics for Arts, Architecture, and Archaeology, edited by Costas Fotakis, Luca Pezzati, Renzo Salimbeni, Proceedings of SPIE Vol. 6618 (SPIE, Bellingham, WA, 2007) Article CID Number.

ISSN 0277-786X

ISBN 9780819467607

Published by

SPIE

P.O. Box 10, Bellingham, Washington $98227-0010$ USA

Telephone +1 3606763290 (Pacific Time) · Fax +1 3606471445

SPIE.org

Copyright (c) 2007, Society of Photo-Optical Instrumentation Engineers

Copying of material in this book for internal or personal use, or for the internal or personal use of specific clients, beyond the fair use provisions granted by the U.S. Copyright Law is authorized by SPIE subject to payment of copying fees. The Transactional Reporting Service base fee for this volume is $\$ 18.00$ per article (or portion thereof), which should be paid directly to the Copyright Clearance Center (CCC), 222 Rosewood Drive, Danvers, MA 01923. Payment may also be made electronically through CCC Online at copyright.com. Other copying for republication, resale, advertising or promotion, or any form of systematic or multiple reproduction of any material in this book is prohibited except with permission in writing from the publisher. The CCC fee code is $0277-786 \mathrm{X} / 07 / \$ 18.00$.

Printed in the United States of America.

Publication of record for individual papers is online in the SPIE Digital Library.

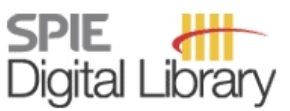

SPIEDigitalLibrary.org

Paper Numbering: Proceedings of SPIE follow an e-First publication model, with papers published first online and then in print and on CD-ROM. Papers are published as they are submitted and meet publication criteria. A unique, consistent, permanent citation identifier (CID) number is assigned to each article at the time of the first publication. Utilization of CIDs allows articles to be fully citable as soon they are published online, and connects the same identifier to all online, print, and electronic versions of the publication. SPIE uses a six-digit CID article numbering system in which:

- The first four digits correspond to the SPIE volume number.

- The last two digits indicate publication order within the volume using a Base 36 numbering system employing both numerals and letters. These two-number sets start with $00,01,02,03,04,05$, $06,07,08,09,0 \mathrm{~A}, 0 \mathrm{~B} \ldots \mathrm{OZ}$, followed by 10-1Z, 20-2Z, etc.

The CID number appears on each page of the manuscript. The complete citation is used on the first page, and an abbreviated version on subsequent pages. Numbers in the index correspond to the last two digits of the six-digit CID number. 


\section{Contents}

ix Conference Committee

\section{KEYNOTE PRESENTATION}

661802 President Kennedy in Dallas: Dealey Plaza memorialized (Keynote Paper) [6618-01]

J. F. Asmus, Univ. of California, San Diego (USA)

\section{OPTICAL COHERENCE TOMOGRAPHY}

661803 Optical coherence tomography for varnish ablation monitoring (Invited Paper) [6618-02] P. Targowski, Nicolaus Copernicus Univ. (Poland); J. Marczak, Military Univ. of Technology (Poland); M. Góra, Nicolaus Copernicus Univ. (Poland); A. Rycyk, Military Univ. of Technology (Poland); A. Kowalczyk, Nicolaus Copernicus Univ. (Poland)

661805 Optical coherence tomography for art conservation and archaeology [6618-04] H. Liang, B. Peric, Nottingham Trent Univ. (United Kingdom); M. Hughes, A. Podoleanu, Univ. of Kent (United Kingdom); M. Spring, The National Gallery (United Kingdom); D. Saunders, The British Museum (United Kingdom)

661806 Optical coherence tomography: non-destructive imaging and spectral information of pigments [6618-05]

G. Latour, Institut des Nanosciences de Paris, CNRS, Univ. Pierre et Marie Curie (France); J. Moreau, Lab. Charles Fabry, Institut d'Optique Graduate School, Univ. Paris Sud, CNRS (France); M. Elias, J.-M. Frigerio, Institut des Nanosciences de Paris, CNRS, Univ. Pierre et Marie Curie (France)

661807 Full-field high-speed optical coherence tomography system for evaluating multilayer and random tissues [6618-06]

I. Gurov, A. Karpets, N. Margariants, E. Vorobeva, St. Petersburg State Univ. of Information Technologies, Mechanics and Optics (Russia)

661808 Optical coherence diagnostics for painting conservation [6618-46]

R. Fontana, M. Bellini, Istituto Nazionale di Ottica Applicata, CNR (Italy); C. Corsi, European Lab. for Non-linear Spectroscopy (Italy); M. Mastroianni, M. Materazzi, L. Pezzati, A. Tortora, Istituto Nazionale di Ottica Applicata, CNR (Italy)

\section{D MODELLING}

661809 Characterisation of the varnish-object interface using white light confocal profilometry (Invited Paper) [6618-07]

W. Wei, Netherlands Institute for Cultural Heritage (Netherlands); J. Frohn, M. Weber, NanoFocus AG (Germany) 
6618 OA X-ray 3D computed tomography of large objects: investigation of an ancient globe created by Vincenzo Coronelli [6618-08]

M. P. Morigi, F. Casali, A. Berdondini, M. Bettuzzi, D. Bianconi, R. Brancaccio, A. Castellani, V. D'Errico, A. Pasini, A. Rossi, Univ. of Bologna (Italy); C. Labanti, Italian National Institute for Astrophysics (Italy); N. Scianna, Univ. of Bologna (Italy)

6618 OC Digitization of art pieces based on 3D, colour, and texture parameters [6618-10] J.-P. Chambard, V. Chalvidan, M. Tazeroualti, HOLO3 (France); F. Larue, J.-M. Dischler, Le Lab. des Sciences de l'Image, de l'Informatique et de la Télédétection (France); V. Vurpillot, A. Legrand, Lab. d'Informatique Graphique et d'Ingénierie de la Vision (France)

6618 OD Cordless hand-held optical 3D sensor [6618-11]

C. Munkelt, C. Bräuer-Burchardt, P. Kühmstedt, I. Schmidt, G. Notni, Fraunhofer-Institute for Applied Optics and Precision Engineering (Germany)

6618 OE Using 3D scanning to monitor wood deformations and to evaluate preservation strategies [6618-12]

M. Brunetti, Istituto per la valorizzazione del legno e delle specie arboree, CNR (Italy);

M. Callieri, Istituto di Scienza e Tecnologie dell'Informazione, CNR (Italy); B. Pizzo, Istituto per la valorizzazione del legno e delle specie arboree, CNR (Italy); C. Montani, Istituto di Scienza e Tecnologie dell'Informazione, CNR (Italy); P. Pingi, Istituto Nazionale di Ottica Applicata, CNR (Italy); R. Scopigno, Istituto di Scienza e Tecnologie dell'Informazione, CNR (Italy)

6618 OF Three-dimensional survey of paint layer [6618-13]

E. Pampaloni, Istituto Nazionale di Ottica Applicata, CNR (Italy); R. Bellucci, Opificio delle Pietre Dure (Italy); P. Carcagni, Istituto Nazionale di Ottica Applicata, CNR (Italy);

A. Casaccia, Opificio delle Pietre Dure (Italy); R. Fontana, M. C. Gambino, R. Piccolo, P. Pingi, L. Pezzati, Istituto Nazionale di Ottica Applicata, CNR (Italy)

6618 OG 3D acquisition and modeling for flint artefacts analysis [6618-14]

B. Loriot, Y. Fougerolle, Le2i Lab., CNRS (France); C. Sestier, CNRS (France); R. Seulin, Le2i Lab., CNRS (France)

$6618 \mathrm{OH}$ Creating A 3D urban model by terrestrial laser scanners and photogrammetry techniques: a case study on the historical peninsula of Istanbul [6618-15]

B. Ergun, Gebze Institute of Technology Geodesy and Photogrammetry (Turkey)

661801 Polarimetry as tool to improve phase measurement in an amplitude modulated laser for submarine archaeological sites inspection [6618-16]

L. Bartolini, L. De Dominicis, M. Ferri de Collibus, G. Fornetti, M. Francucci, M. Guarneri, E. Paglia, C. Poggi, R. Ricci, ENEA, Italian National Agency for New Technologies, Energy and Environment (Italy)

6618 0J Simultaneous acquisition of 3D shape and color texture from large frescoed areas [6618-37]

M. Caldon, M. Fedel, L. Poletto, INFM, Univ. of Padua (Italy); N. Brusco, G. Cortelazzo, A. Paviotti, Univ. of Padua (Italy) 
6618 OK Volumetric 3D display for visualization of archaeological samples investigated with neutron tomography [6618-41]

V. Fiasconaro, F. Andreoli, M. Palomba, A. Papaleo, R. Rosa, ENEA (Italy)

\section{SPECTROSCOPIC TECHNIQUES}

$66180 \mathrm{M}$ Multi-spectral fluorescence imaging for cultural heritage (Invited Paper) [6618-17]

D. Comelli, G. Valentini, R. Cubeddu, IFN-CNR, Politecnico di Milano (Italy); L. Toniolo, Politecnico di Milano (Italy)

$6618 \mathrm{OP}$ Laser-induced fluorescence imaging for studies of cultural heritage [6618-20]

R. Grönlund, S. Svanberg, J. Hällström, K. Barup, Lund Univ. (Sweden); G. Cecchi, V. Raimondi, D. Lognoli, L. Palombi, Institute for Applied Physics Nello Carrara (Italy)

$66180 Q \quad$ Non-destructive detection of lead glass alteration by reflectance spectroscopy [6618-21] M. Elias, Institut des NanoSciences de Paris, Univ. Pierre et Marie Curie, CNRS (France); C. Charbonneau, Ctr. de Recherche et de Restauration des Musées de France, CNRS (France); J. M. Frigerio, Institut des NanoSciences de Paris, Univ. Pierre et Marie Curie, CNRS (France)

6618 OR Effects of gamma irradiation on the colour of pigments [6618-40] D. C. Negut, C. C. Ponta, R. M. Georgescu, I. V. Moise, Horia Hulubei National Institute of R\&D for Physics and Nuclear Engineering (Romania); Gh. Niculescu, National Research Institute for Conservation and Restoration of Movable National Cultural Heritage (Romania); A. I. M. Lupu, National Art Museum of Romania (Romania)

6618 OS A Bayesian decision model for watercolour analysis [6618-36] V. Kokla, A. Psarrou, V. Konstantinou, Univ. of Westminster (United Kingdom)

6618 OT Color appearance of painted artworks through protective glass [6618-38] A. Farini, R. Arrighi, C. Gheri, Istituto Nazionale di Ottica Applicata, CNR (Italy); M. L. Grilli, A. Krasilnikova Sytchkova, A. Piegari, ENEA (Italy)

6618 OU Laser induced breakdown spectroscopy in paintings and sculptures research [6618-47] A. Sarzyński, W. Skrzeczanowski, J. Marczak, Military Univ. of Technology (Poland)

6618 OV Physical phenomena disturbing LIBS analysis [6618-49]

A. Sarzyński, W. Skrzeczanowski, J. Marczak, Military Univ. of Technology (Poland)

\section{COHERENT TECHNIQUES}

$66180 \mathrm{~W}$ Multifunctional encoding system for assessment of movable cultural heritage (Invited Paper) [6618-22]

V. Tornari, E. Bernikola, Foundation for Research and Technology-Hellas (Greece);

W. Osten, R. M. Groves, Univ. Stuttgart (Germany); G. Marc, Ctr. Spatial de Liège (Belgium); G. M. Hustinx, OPTRION (Belgium); E. Kouloumpi, National Gallery-Alexandros Soutzos Museum (Greece); S. Hackney, Tate (United Kingdom) 
6618 OY Defect detection of wall paintings in the Château de Versailles using TV-holography and IR thermography [6618-24]

J.-P. Chambard, HOLO3 (France); A. Roche, Lab. d'Analyses et de Recherche pour la Conservation et la Restauration d'Oeuvres d'Art (France)

$66180 Z$ Development of impact assessment procedure and preliminary results with digital holographic speckle pattern interferometry for signatures multi-encoding use [6618-25] E. Bernikola, K. Hatzigiannakis, Y. Orphanos, Foundation for Research and TechnologyHellas (Greece); E. Kouloumpi, National Gallery-Alexandros Soutzos Museum (Greece); V. Tornari, Foundation for Research and Technology-Hellas (Greece)

661810 Shearography as part of a multi-functional sensor for the detection of signature features in movable cultural heritage [6618-26]

R. M. Groves, W. Osten, Univ. Stuttgart (Germany); M. Doulgeridis, E. Kouloumpi, National Gallery, Alexandros Soutzos Museum (Greece); T. Green, S. Hackney, Tate (United Kingdom); V. Tornari, Foundation for Research and Technology-Hellas (Greece)

661811 Explosive embossing of holographic structures [6618-27]

T. Scholz, J. Seewig, E. Reithmeier, Leibniv Univ. Hannover (Germany); G. Helferich, Fraunhofer-Institut für Chemische Technologie (Germany)

661812 Role of dynamic holography with photorefractive crystals in a multi-functional sensor for the detection of signature features in movable cultural heritage [6618-28]

C. Thizy, M. Georges, Univ. de Liège (Belgium); M. Doulgeridis, E. Kouloumpi, National Gallery, Alexandro Soutzos Museum (Greece); T. Green, S. Hackney, Tate (United Kingdom); V. Tornari, Foundation for Research and Technology-Hellas (Greece)

IMAGING

661813 Multi-spectral IR reflectography (Invited Paper) [6618-29]

R. Fontana, D. Bencini, P. Carcagni, M. Greco, M. Mastroianni, M. Materazzi, E. Pampaloni, L. Pezzati, Istituto Nazionale di Ottica Applicata, CNR (Italy)

661815 PRISMS: a portable multispectral imaging system for remote in situ examination of wall paintings [6618-31]

H. Liang, K. Keita, T. Vajzovic, Nottingham Trent Univ. (United Kingdom)

661816 Multispectral imaging of paintings: instrument and applications [6618-32]

C. Bonifazzi, Univ. of Ferrara (Italy); P. Carcagnì, A. Della Patria, R. Fontana, M. Greco, M. Mastroianni, M. Materazzi, E. Pampaloni, L. Pezzati, Istituto Nazionale di Ottica Applicata, CNR (Italy); A. Romano, Univ. of Ferrara (Italy)

661817 NDT methods in artwork corrosion monitoring [6618-35]

D. Ambrosini, A. Paoletti, D. Paoletti, S. Sfarra, DIMEG, Univ. of L'Aquila (Italy) 


\section{LASER CLEANING}

$66181 \mathrm{C}$ Batory's Chapel at Wawel Castle, Cracow: laser cleaning and hue measurements of epitaph and stalls [6618-44]

J. Marczak, Military Univ. of Technology (Poland); A. Koss, Academy of Fine Arts in Warsaw (Poland); R. Ostrowski, A. Rycyk, M. Strzelec, Military Univ. of Technology (Poland)

6618 1D Laser damage thresholds of bone objects [6618-45]

R. Ostrowski, J. Marczak, M. Strzelec, Military Univ. of Technology (Poland); A. Koss,

Academy of Fine Arts (Poland)

\section{NOVEL OPTICAL TECHNIQUES}

$66181 \mathrm{~F}$ The relationship between surface incline and con-focal chromatic aberration sensor response [6618-48]

J. W. McBride, P. J. Boltryk, Z. Zhao, Univ. of Southampton (United Kingdom)

6618 1G A versatile microfadometer for lightfastness testing and pigment identification [6618-33]

A. Lerwill, Tate (United Kingdom) and Nottingham Trent Univ. (United Kingdom);

J. H. Townsend, Tate (United Kingdom); H. Liang, Nottingham Trent Univ. (United Kingdom);

S. Hackney, J. Thomas, Tate (United Kingdom)

$66181 \mathrm{H}$ Petrographic microscope investigation of mortar and ceramic technologies for the conservation of the built heritage [6618-34]

S. Pavia, Trinity College Dublin (Ireland); S. Caro, Museo Paleontologico de Enciso (Spain)

Author Index 
Downloaded From: https://www.spiedigitallibrary.org/conference-proceedings-of-spie on 26 Apr 2023

Terms of Use: https://www.spiedigitallibrary.org/terms-of-use 


\title{
Conference Committee
}

\author{
Symposium Chairs
}

Wolfgang Osten, Universität Stuttgart (Germany)

Malgorzata Kujawinska, Warsaw University of Technology (Poland)

Ralph P. Tatam, Cranfield University (United Kingdom)

Conference Chairs

Costas Fotakis, Foundation for Research and Technology-Hellas

(Greece)

Luca Pezzati, National Institute of Applied Optics/CNR (Italy)

Renzo Salimbeni, Institute of Applied Physics N. Carrara/CNR (Italy)

Program Committee

John F. Asmus, University of California, San Diego (USA)

Marta Castillejo, Spanish Council for Scientific Research (Spain)

Alberto de Tagle, Netherlands Institute for Cultural Heritage (Netherlands)

Alexander J. Kossolapov, The Hermitage Museum (Russia)

Michel Menu, Ctr. de Recherche et des Restauration des Musees (France)

David R. Saunders, National Gallery, The British Museum (United Kingdom)

John M. Taylor, National Research Council Canada (Canada)

Session Chairs

Keynote Presentation

Luca Pezzati, National Institute of Applied Optics/CNR (Italy)

Optical Coherence Tomography

Costas Fotakis, Foundation for Research and Technology-Hellas

(Greece)

John F. Asmus, University of California, San Diego (USA)

3D Modelling

Piotr Targowski, Nicolaus Copernicus University (Poland)

Haida Liang, Nottingham Trent University (United Kingdom)

Spectroscopic Techniques

Andreas Burmester, Doerner Institut (Germany) 
Coherent Techniques

Igor P. Gurov, St. Petersburg State University of Information

Technologies, Mechanics and Optics (Russia)

Imaging

Andreas Burmester, Doerner Institut (Germany)

Laser Cleaning

Costas Fotakis, Foundation for Research and Technology-Hellas

(Greece)

Novel Optical Techniques

Renzo Salimbeni, Istituto di Fisica Applicata N. Carrara, CNR (Italy) 